\title{
Article \\ Combining Chemical Functionalization and FinFET Geometry for Field Effect Sensors as Accessible Technology to Optimize pH Sensing
}

\author{
Dipti Rani ${ }^{1}$, Serena Rollo ${ }^{1,2}$, Wouter Olthuis ${ }^{2}$ (D) , Sivashankar Krishnamoorthy ${ }^{1}$ (D) and César Pascual García ${ }^{1, *}$ \\ 1 Nano-Enabled Medicine and Cosmetics Group, Materials Research and Technology Department, \\ Luxembourg Institute of Science and Technology (LIST), L-4422 Belvaux, Luxembourg; \\ dipti.rani@list.lu (D.R.); serena.rollo@list.lu (S.R.); sivashankar.krishnamoorthy@list.lu (S.K.) \\ 2 BIOS Lab on Chip Group, MESA+ Institute for Nanotechnology, University of Twente, 7522 NB Enschede, \\ The Netherlands; w.olthuis@utwente.nl \\ * Correspondence: cesar.pascual@list.lu; Tel.: +352-275-888-583
}

Citation: Rani, D.; Rollo, S.; Olthuis, W.; Krishnamoorthy, S.; Pascual García, C. Combining Chemical Functionalization and FinFET Geometry for Field Effect Sensors as Accessible Technology to Optimize pH Sensing. Chemosensors 2021, 9, 20 https://doi.org/10.3390/

chemosensors 9020020

Academic Editor: Simas Rackauskas Received: 7 December 2020

Accepted: 15 January 2021

Published: 21 January 2021

Publisher's Note: MDPI stays neutral with regard to jurisdictional claims in published maps and institutional affiliations.

Copyright: (c) 2021 by the authors. Licensee MDPI, Basel, Switzerland. This article is an open access article distributed under the terms and conditions of the Creative Commons Attribution (CC BY) license (https:// creativecommons.org/licenses/by/ $4.0 /)$.

\begin{abstract}
Field Effect Transistors (FETs) have led the progress of applications measuring the acidity in aqueous solutions thanks to their accuracy, ease of miniaturization and capacity of multiplexing. The signal-to-noise ratio and linearity of the sensors are two of the most relevant figures of merit that can facilitate the improvements of these devices. In this work we present the functionalization with aminopropyltriethoxysilane (APTES) of a promising new FET design consisting of a high height-towidth aspect ratio with an efficient 2D gating architecture that improves both factors. We measured the transistor transfer and output characteristics before and after APTES functionalization, obtaining an improved sensitivity and linearity in both responses. We also compared the experimental results with a site-biding model of the surface buffering capacity of the APTES functionalized layers.
\end{abstract}

Keywords: ISFET; FinFETs; nanowire; APTES; pH sensing

\section{Introduction}

In 1970, Bergveld invented the ion sensitive field effect transistor (ISFET) using a variation of a metal oxide field effect transistor (MOSFET) that substituted the metallic gate by an electrolyte connected to a reference electrode [1]. The device was sensitive to the charges adsorbed at the oxide barrier. Since then, different adaptations of this transistor have been proposed as chemical sensors under the generic denomination of CHEMFETs [2]. The first research with ISFETs focused on the measurement of physiological acidity [3] and as a result of these studies, many of today's $\mathrm{pH}$ sensors in research and commercial applications are ISFETs. At the present moment, the investigations on pH-ISFETs continue to grasp a lot of interest for enzyme field effect transistors (ENFETs) and microfluidic setups like the one that monitors the action of the Polymerase Chain Reaction (PCR) for gene sequencing $[4,5]$. ISFET $\mathrm{pH}$ sensors are particularly well-suited for such applications because of their ease of integration with readout and complementary metal oxide semiconductor (CMOS) circuits that allow miniaturization and massive multiplexing [6,7]. The progress in the research of these applications has been boosted because the semiconductor industry is able to offer a wide access to sophisticated ISFETs. Commercially available unmodified CMOS circuits or extended gate configurations can deliver hundreds of thousands of miniaturized $\mathrm{pH}$ sensors for monitoring the acidity at low costs [8]. A limiting factor with indirect gating is the parasitic interferences introduced by the capacitive divisions introduced at the transistor input with the external gate that affect the signal-to-noise ratio, in configurations with several multiplexed devices. While the signal of $\mathrm{pH}$-ISFET sensors can be enhanced by capacitive coupling with backgating used to amplify the signal [9], ultimately this does not improve the resolution of $\mathrm{pH}$, as the noise is also amplified. Architectures using gating configurations directly in contact with both the electrolyte and the semiconductor channel 
have the potential to increase the signal-to-noise ratio and contribute to the miniaturization of $\mathrm{pH}$ sensors.

Nano-FETs, and particularly nanowires (NWs), are at the forefront of research being preferred over planar sensors for the detection of complex biomolecules like deoxyribonucleic acid (DNA) and proteins that have lower diffusion rates than protons [10]. The smaller size of NWs offers higher sensitivities to fewer molecules than bigger traditional sensors, as well as lower limits of detection. It is to be noted that the improvements of limits of detection for proteins and nanowires is based on the advantages of the small size of NWs for the diffusion limited processes that play a central role given the ultralow concentrations at which these molecules can be expected in clinical applications [11]. In addition to this aspect linked to the diffusion, which has a lower benefit for sensing fast-moving protons, NWs have other advantages relevant for $\mathrm{pH}$ monitoring. In particular, the three-dimensional gating offers a larger surface-to-volume ratio improving directly the signal-to-noise ratio and the smaller footprint that benefits the miniaturization [12]. The fabrication of nano ISFETs initially required sub $100 \mathrm{~nm}$ lithography. However, using combinations of optical lithography and wet etching that take advantage of the anisotropic properties of crystalline silicon, it is possible to bring the lateral resolution down to a size comparable to the depletion width induced by the $\mathrm{pH}$ changes. Thus, optically lithographed nanowires can become very sensitive $[13,14]$. These fabrication processes are also accessible because the dielectric gate can be achieved without the use of large resources using thermal oxidation of silicon to obtain an $\mathrm{SiO}_{2}$ dielectric barrier, where protons are adsorbed and sensed. One relevant downside of NWs, for highly multiplexed configurations, is that they have lower currents (in the order of tenths of $\mathrm{nA}$ ) than other transistors requiring more sophisticated measuring apparatus. For a detailed comparison of different ISFET technologies we suggest a recent review by Moser and co-workers [7].

Regarding sensing materials, $\mathrm{SiO}_{2}$ has limitations due to the decrease in sensitivity at acidic pHs and drift [15] that can also be overcome with chemical functionalization, which is cost-efficient for research and prototyping. The acidic saturation of $\mathrm{SiO}_{2}$ that limits the linear $\mathrm{pH}$ response occurs because the surface charging is fully dominated by the dissociation of single coordinated silanol groups (with dissociation constant $\mathrm{pK}_{\mathrm{a}} \approx 6$ ), since the dissociation constant corresponding to the protonation of these groups is too low $\left(\mathrm{pK}_{\mathrm{b}} \approx-2\right)[16]$. The linear dynamic range and the isolation of the dielectric barrier of $\mathrm{SiO}_{2}$ to other ions can be improved by changing the surface chemistry with self-assembled monolayers that change the nature or the chemical groups adsorbing protons. The linkage of 3-aminopropyltriethoxysilane (APTES) at the $\mathrm{SiO}_{2}$ /liquid interface changes the silanol groups by amino groups that can accept and release protons. APTES functionalization has been reported to improve the linear range of $\mathrm{pH}$ sensing [17] and it is also employed as a first step for the functionalization of receptors for detecting DNA and proteins. APTES has also shown an improved stability over time and cycles of $\mathrm{pH}$ [18]. The APTES silanization can be performed by solution-phase or vapor-phase methods, although liquid-phase silanization of APTES requires a strict control over the anhydrous phase to achieve uniform layers. Moreover, the inhomogeneities happening during the functionalization of nanosensors is critical as they result in large variabilities among devices due to the differences in surface coverage that occur in the same length scales of the NWs and which can be also triggered by the topological profile of the nanosensors.

Recently, we presented an FinFET configuration consisting of a high height-to-width aspect ratio $(>10)$ geometry in which the sensor width was comparable to that of the NWs [19]. This configuration offers a larger surface area available for functionalization than the one of NWs, and it is less sensitive to defects due to the larger conduction channel. Yet, the high aspect ratio of FinFET has the same benefits of the surface area-to-volume ratio as that of NWs, offering also the advantage of three-dimensional gating and the same dynamic range for NWs and FinFETs of the same width. In a high aspect ratio FinFET, the total current is enhanced by the same factor as the geometrical ratio. For example, in a FinFET with a ratio of ten between the height and the width of the device, the current 
is also ten times higher than in an NW with the same $\mathrm{pH}$ sensitivity [19]. In addition, while the gate-geometry of NWs results in a non-linear response of the output current, in particular close to the threshold voltage, in the case of high aspect ratio FinFETs the depletion is induced almost entirely in the direction perpendicular to the FinFET plane, and thus they offer an increase of linearity in the output current, which could be then used to simplify the measuring circuits with respect to the traditional configuration that uses the compensation of the surface potential with the reference electrode, and which may be inconvenient in highly multiplexed systems [20].

In this work, we show chemically modified p-type high aspect-ratio Si FinFETs with an $\mathrm{SiO}_{2}$ dielectric barrier modified with a vapor-phase silanization of APTES molecules done in a home-built setup. The FinFETs are characterized before and after the APTES silanization process in different $\mathrm{pH}$ buffer solutions. We study the $\mathrm{pH}$ sensitivities of $\mathrm{Si}$ FinFETs associated with the surface potential as a function of buffer $\mathrm{pH}$ change. We quantify the APTES molecules bound at the oxide surface of FinFETs that contributed to the increase in sensitivity and linearity in $\mathrm{pH}$ response. We used a multi-parametric surface plasmon resonance (MP-SPR) setup to evaluate the thickness of the APTES layer and the coverage of molecules bound at the sensor surface $\left(\mathrm{SiO}_{2}\right.$ coated SPR chips) using LayerSolver software. We compared also the experimental buffering capacity of our system with a site-biding model using the number of APTES groups obtained by SPR. We measured a small increase in the $\mathrm{pH}$ sensitivity and in the linearity of the FinFETs response after APTES silanization. The improvement of the linear response was also observed in the output response of the FinFET. The method presented here is promising for the development of reliable bio-FETs as it can be extended to subsequent biomolecule immobilization steps to have a quality check of the surface functionalization process and to implement necessary further modifications.

\section{Materials and Methods}

\subsection{FinFETs Fabrication}

The fabrication of Si FinFETs was reported in our previous work by Rollo et al. [19], as summarized in Figure 1a. The FinFETs were fabricated using anisotropic wet-etching on p-type Silicon on insulator (SOI) (Ultrasil Corporation, Hayward, CA, USA) having a $2.2 \pm 0.1 \mu \mathrm{m}$ thick silicon device layer $(<110>$ oriented) with conductivity of $0.115 \Omega \cdot \mathrm{cm}$ (equivalent doping $\sim 10^{17} \mathrm{~cm}^{-3}$ ) and a $1 \mu \mathrm{m}$ thick buried $\mathrm{SiO}_{2}$. The wafers were diced into $1 \times 1 \mathrm{~cm}^{2}$ chips. A $50 \mathrm{~nm}$ layer of $\mathrm{SiO}_{2}$ was grown on top of the $\mathrm{Si}$ device layer by thermal oxidation performed at $1050{ }^{\circ} \mathrm{C}$ in $\mathrm{O}_{2}$ environment $(200 \mathrm{sccm})$ for $300 \mathrm{~s}$. The devices in this work were patterned using electron-beam (e-beam) lithography by first spin-coating the resist over the SOI chips and exposing them on an adapted electron microscope (FEI Helios). However, we have also shown that these devices can be made using optical lithography, thus they can be accessible to labs with no facilities for direct nanolithography [21]. We used the FinFET design with a $<110>$ crystallographic orientation along the longitudinal axis of the FinFET, that was transferred onto the underlying oxide using reactive ion etching process. Following the lithography, a short hydrofluoric acid etching step was carried out to remove the unwanted oxide outside the masked regions obtaining a smooth Si surface prior to the process of anisotropic etching. A water solution of $25 \%$ tetramethylammonium hydroxide (TMAH) with $8.5 \%$ vol. isopropanol was used to etch the Si device layer anisotropically obtaining vertical walls on the FinFET sensing regions. A $20 \mathrm{~nm}$ layer of $\mathrm{SiO}_{2}$ was thermally grown over the patterned $\mathrm{Si}$ device layer as the dielectric barrier of the FET. Ohmic contacts and metallic leads were defined using mask-less laser lithography and e-beam evaporation. The contacts and the chip surface were passivated using an SU8 epoxy layer leaving open the FinFET region. For the measurements in liquid, the chips were wire bonded on home-designed printed circuit boards and encapsulated using medical-grade epoxy. 


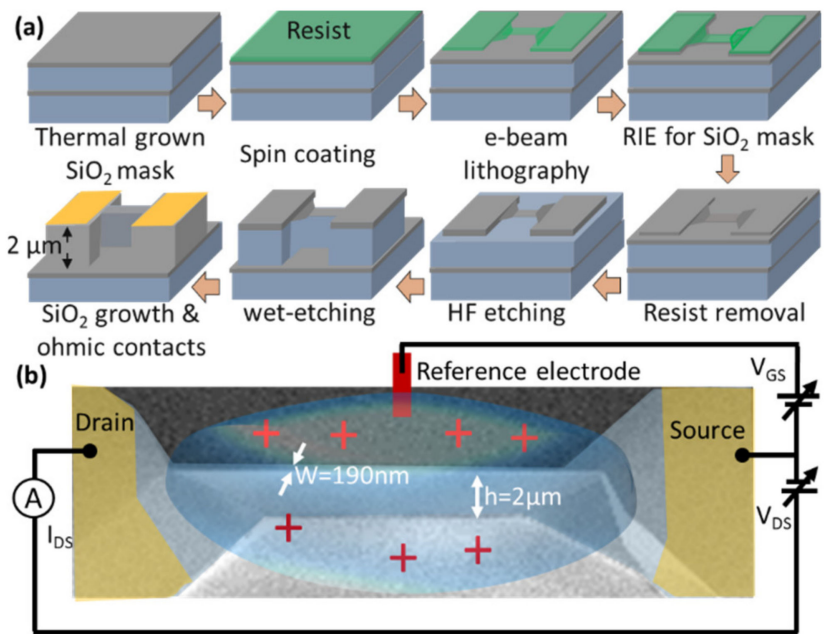

Figure 1. (a) Schematics of the process flow for the fabrication of Si Fin-field effect transistors (FETs). Silicon, $\mathrm{SiO}_{2}$, e-beam sensitive resist, and ohmic contacts are represented in light blue, grey, green and yellow, respectively. (b) Schematics illustrating operation of Si FinFET in liquid gate configuration.

\subsection{Chemical Modification of FinFETs and SPR Sensors: Vapor-Phase APTES Silanization}

The chemical modification of the FinFET devices started with surface activation of the hydroxyl groups on the sensing $\mathrm{SiO}_{2}$ surfaces by low ozone exposing the chips into an UV lamp chamber for $5 \mathrm{~min}$. Immediately after, the silanization process was carried out at a low-pressure atmosphere $\left(\mathrm{p}=1 \times 10^{-3} \mathrm{mBar}\right)$ inside a desiccator where small volumes (1-2 mL) of APTES were placed in a glass vial along with the FinFET chips (as shown in Figure 2a). The silanization process was carried out for $2.5 \mathrm{~h}$ at room temperature. After the functionalization, the chips were thoroughly rinsed with isopropanol to remove any excess silane molecules and blow dried with nitrogen. The silanization protocol was simultaneously implemented on SPR sensor chips to have a similar density of APTES molecules (Figure $2 b$ ).
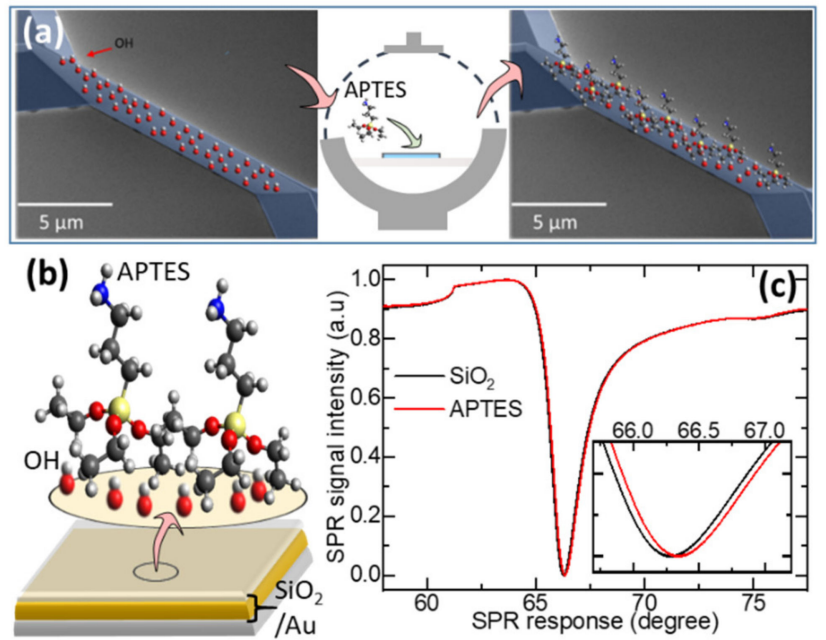

Figure 2. (a) Schematic representation of the Fin-FET surface before and after the vapor-phase 3aminopropyltriethoxysilane (APTES) silanization. (b) Schematics illustrating the chemically modified SPR sensor chip with APTES layer. A $10 \mathrm{~nm} \mathrm{SiO}_{2}, 50 \mathrm{~nm}$ Au SPR layer was used for exploring the APTES functionalization. (c) Full SPR reflectance before (in black) and after the APTES silanization process (in red) at $\lambda=785 \mathrm{~nm}$. The inset shows the shift in SPR angular peak minimum $\left(\Theta_{S P R}\right)$. 


\section{3. pH Measurements Using FinFETs}

The encapsulated FinFET chips were operated in liquid gate configuration using an external reference electrode (DriRef- $2 \mathrm{Ag} / \mathrm{AgCl}$ from WPI) in buffers consisting of a mixture of $\mathrm{KH}_{2} \mathrm{PO}_{4}$, citric and boric acids at $0.1 \mathrm{M}$ all, mixed in equal volumes with $0.1 \mathrm{M}$ $\mathrm{KNO}_{3}$ solution. For the $\mathrm{pH}$-sensing measurements with FinFETs, the $\mathrm{pH}$ of the prepared buffer solution was varied in the range of 3 to 11 by adding required volumes of $0.1 \mathrm{M}$ $\mathrm{KOH}$. A Keithley $2614 \mathrm{HB}$ DC source meter was used to apply the voltage to the source and drain and the reference electrode. To characterize multiple FinFETs in sequence, a multiplexer Keithley 3706A System Switch/Multimeter connected to a switching box was used. The devices were characterized before and after APTES functionalization.

\subsection{MP-SPR Measurements}

MP-SPR measurements were performed using an SPR model Navi 220A ${ }^{\mathrm{TM}}$ (BioNavis Ltd., Tampere, Finland) setup. The SPR setup used two incident laser wavelengths, 670 and $785 \mathrm{~nm}$, inlet and outlet (waste) tubings, and two independent flow channels. We used SPR sensor chips with a thin layer of $\mathrm{SiO}_{2}$ above the gold layer (from BioNavis Ltd.) for the APTES silanization to have binding reactions like the FinFET sensor surface. The temperature was kept at $20^{\circ} \mathrm{C}$ and the measurements were carried out in Milli-Q water.

SPR allows measuring the layer thickness even with submonolayer sensitivity following the changes in refractive index of the layers in contact with the SPR sensor chips detected as changes in the angle of minimum reflectivity $\left(\Theta_{S P R}\right)$. MP-SPR uses more than one wavelength in combination with the scanning angle to obtain the layer thickness, refractive indices, and surface mass densities $[22,23]$. Figure $2 b$ illustrates the different layers on the SPR sensor chips, and a thin layer of $\mathrm{SiO}_{2}$ above the gold $(\mathrm{Au})$ layer was used to adapt for the APTES silanization to yield binding reactions equivalent to the ones occurring during FinFET functionalization. We evaluated the layer thickness $(d)$ and refractive index (n) for APTES molecules bound on sensor surface using the SPR Navi LayerSolver v. 1.4 (BioNavis Ltd., Tampere, Finland) software using the full SPR angular reflectance measured at two wavelengths (670 and $785 \mathrm{~nm}$ ), at the same point of time. The LayerSolver software creates a model of the different layers on the sensor chip to match the full reflectance on the SPR sensor chip to adjust the change of $\Theta_{S P R}$ at the two wavelengths fitting the refractive index $(n)$ and thickness $(d)$ corresponding to the APTES layer using Fresnel refraction laws (the details are shown in Supplementary Materials). The obtained $n$ and $d$ are then connected to the mass surface concentration of adsorbed APTES $(\Gamma)$ by de Feijter model that assumes that the refractive index is a linear function of the concentration $(C)$ over a wide range of concentrations:

$$
\Gamma=d \frac{n-n_{0}}{\frac{d n}{d C}}
$$

where $n_{0}$ is the medium refractive index and $d n / d C$ is a coefficient known as refractive index increment, which is the derivative of the refractive index with respect to the molecule concentration in solution. When $d n / d C$ is not available from experimental data, it can be also estimated from the physicochemical properties of APTES, in particular from the volumetrical density $v$ and the refractive indexes from the solvent and APTES [24]:

$$
\frac{d n}{d C}=\frac{3}{2} n_{0} v \frac{n^{2}-n_{0}^{2}}{n^{2}+2 n_{0}^{2}}
$$

\section{Results}

We deployed MP-SPR to evaluate the thickness and molecular density of the APTES layer deposited via vapor-phase silanization. Figure $2 \mathrm{c}$ shows an exemplary measurement taken at $\lambda=785 \mathrm{~nm}$ before (black curve) and after the APTES silanization (red curve) also zoomed in the inset. As shown, on binding of APTES molecules at the SPR chip, $\Theta_{S P R}$ shifts towards larger angles [20]. We obtained the thickness and refractive index of APTES layer as $d=1.7 \mathrm{~nm}$ and $n=1.41$. Parallel to the chip functionalisation on the FinFETs, we introduced 
a chip $\mathrm{SiO}_{2} / \mathrm{Si}$ that was grown in the same process as our devices. The thickness measured by SPR was comparable to the values obtained from ellipsometry $(d=1.9 \pm 0.2 \mathrm{~nm})$ carried out on APTES-coated $\mathrm{SiO}_{2} / \mathrm{Si}$ chips (details in Supplementary Materialsand was higher than the APTES monolayer thickness reported in literature $(\sim 1 \mathrm{~nm})$ [25]. As the thickness and refractive index measured via SPR gives an average over the entire sensor surface, we assumed that the higher thickness of APTES layer could be due to partial agglomerates that formed on the sensor surface during the vapour-phase silanization process. We used the specific volume value of APTES from the literature and Equation (1) to evaluate the surface coverage of APTES molecules at the sensor surface $\left(\mathrm{N}_{\mathrm{s}}\right)$ as $4.45 \times 10^{18}$ molecules $/ \mathrm{m}^{2}$. As we do not have a way to determine the distribution of agglomerates, we assumed this number as the number of APTES group present on the sensor for our modelling to calculate the sensitivity of the ISFETs.

\subsection{Modification of the $p H$ Sensitivity of the Surface Potential with APTES}

The experiments for $\mathrm{pH}$ sensing were carried out in three devices using a liquid gate configuration as shown in Figure $1 \mathrm{~b}$. The working ranges of these devices were characterized in our previous work [19]. We characterized the changes in surface potential due to the chemical modification of the FinFETs following the $V_{G S}$ at fixed $I_{D S}$ with different $\mathrm{pH}$ buffers (in a range from 11 to 3), measured before and after APTES functionalization. Figure 3 reports the results of $V_{G S}$ vs. $I_{S D}$ from a representative FinFET at different $\mathrm{pHs}$ (3, $5,7,8,10)$ before and after APTES silanization. For these measurements, $V_{D S}$ was fixed at $300 \mathrm{mV}$. The dimensions of this device (hereby designed as device 1) characterized by SEM microscopy were $190 \mathrm{~nm}, 14$, and $2 \mu \mathrm{m}$ for width, length and height, respectively. We compared the changes of $V_{G S}$ with $\mathrm{pH}$ on two other devices of the same batch hereby designated devices 2 and 3, which had bigger widths of 300 and $320 \mathrm{~nm}$, respectively. In all devices we observed that the $I_{D S}$ transfer characteristics shifted towards more positive $V_{G S}$ values with more alkaline solutions due to the effect of the adsorbed charges on the majority charge carriers within the channel (holes in our case), as illustrated on Figure 3a with the data from device 1 having the smallest width. At higher $\mathrm{pH}$ values there are fewer protons in the solution and therefore the equilibrium with the surface chemical groups promotes deprotonation, resulting in the negatively charged oxide. This change in the surface potential $\left(\psi_{0}\right)$ influences the hole carriers within the FinFET channel, causing the need for the modulation of the electrolyte potential $\left(\Delta V_{G S}=-\Delta \psi_{0}\right)$ to keep the current constant through the channel. $V_{G S}$ vs $\mathrm{pH}$ extracted at constant $I_{D S}=2.25 \mu \mathrm{A}$ before (black solid squares) and after the APTES silanization (red open circles) is shown as a scatter plot in Figure $3 \mathrm{~b}$. We calculated the average sensitivity of the oxide surface $\left(\Delta V_{G S} / \Delta p H\right)$ before and after APTES silanization process from a linear fit in Figure $3 \mathrm{~b}$. The $\Delta V_{G S} / \Delta p H$ of bare $\mathrm{SiO}_{2}$ was $31 \pm 1 \mathrm{mV} / \mathrm{pH}$ with a relatively non-linear response calculated by the coefficient of the determination R-squared $\left(R_{s q}\right)$ calculated from the fitting $R_{s q}=0.988$. The sensitivity value is comparable to the values that we obtained in similar devices where we used the same oxidation process [21]. As expected, the values that deviated most from the linear behavior were the values at the most acidic $\mathrm{pH}$ 's. The non-linearity in the $\mathrm{pH}$ response for $\mathrm{SiO}_{2}$-based dielectrics has been previously explained in literature and is linked to the low the protonation affinity of silanol groups $\left(p K_{b} \approx-2\right)$ that makes the proton dissociation the only process associated to the charging of the surface [16]. A relatively higher linearity with $R_{s q}=0.996$ and average $\Delta V_{G S} / \Delta p H=36 \pm 1 \mathrm{mV} / \mathrm{pH}$ was observed for the APTES functionalized surfaces. This was a modest improvement, but it was consistent with other works in literature $[17,18]$. As APTES has a double charging mechanism corresponding to the active protonation and deprotonation of amino groups that have $p K_{a}$ and $p K_{b}$ of 9 [17] and 3.63 [26], respectively, the increase linearity was expected. Also, the narrower gap between $p K_{a}$ and $p K_{b}$ contribute to the higher sensitivity. 

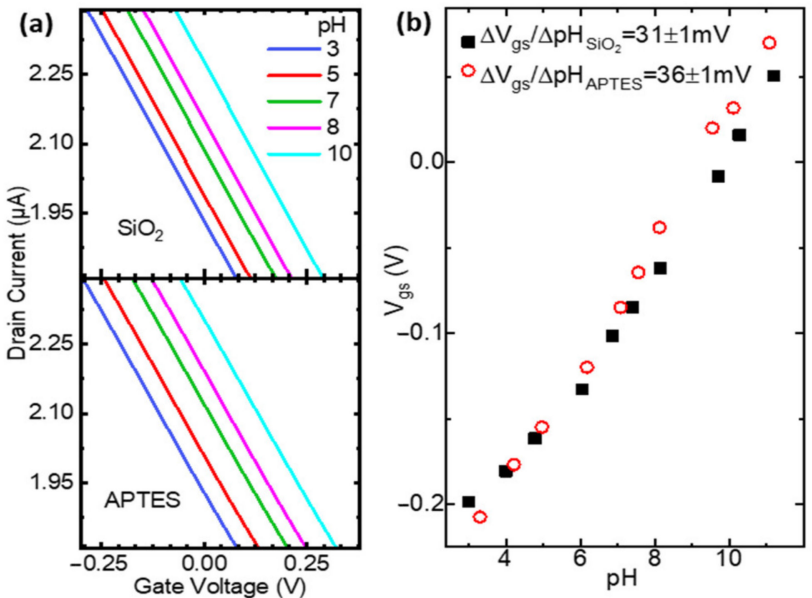

Figure 3. (a) Transfer $I_{D S}$ vs. $V_{G S}$ of a FinFET before (top) and after the APTES silanization process (bottom) with different $\mathrm{pH}(3,5,7,8,10)$ shown in different colors. (b) Scatter plot of $V_{G S}$ vs. $\mathrm{pH}$ before (black squares) and after the APTES silanization process (red open circles). The $\Delta V_{G S} / \Delta \mathrm{pH}$ evaluated from the linear fit of the plots are indicated in the inset. For these measurements $V_{D S}$ was fixed at $300 \mathrm{mV}$.

\subsection{Chemical Characterisation of the Surface Properties Using the Buffering Capacity Determined} from the Surface Potential

To get a better insight on the properties of the current APTES functionalization we compared experimental results with a site binding model of the dielectric surface. The relation between the changes in the surface potential $\left(\psi_{0}\right)$ with the $\mathrm{pH}$ of the bulk solution are compensated by $V_{G S}\left(\Delta V_{G S}=-\Delta \psi_{0}\right)$. The sensitivity of ISFETs for $\psi_{0}$ is well described in literature [16] with a Nernstian framework:

$$
\frac{\Delta \psi_{0}}{\Delta p H_{B}}=-2.303 \frac{k T}{q} \alpha
$$

where $k, T$ and $q$ are the Boltzmann constant, absolute temperature and elementary charge, respectively. $\alpha$ is a dimensionless sensitivity parameter between 0 and 1 determined by the electrostatic interactions at the surface of the dielectric and distribution of ions inside the electrolyte, and is given by [16]:

$$
\alpha=\frac{1}{1+2.3 \frac{k T C_{\text {diff }}}{q^{2} \beta_{\text {int }}}}
$$

where $C_{\text {diff }}$ is the differential capacitance of the electrical double layer that depends upon the properties of electrolyte solution. The intrinsic buffer capacity $\left(\beta_{\text {int }}\right)$ gives the measure of charging capability of the oxide surface with changes in $\mathrm{pH}$ of the buffer solution. It depends on the intrinsic properties of the oxide surface and changes every time a chemical group contributes to the charging and un-charging of the sensor interface. In a FET device, for low $C_{\text {diff, }}$, and high $\beta_{\text {int }}, \alpha$ will be close to 1 and thus maximum $\mathrm{pH}$ sensitivity can be obtained. For $C_{\text {diff }}$ and $\beta_{\text {int }}$ we used a site binding model combined with a Stern approximation previously used in literature [16]. In this model $C_{\text {diff }}$ is a combination of the Gouy-Chapman approximation $\left(C_{G C h}\right)$ with an estimation of the stern layer $\left(C_{S t e r n}=0.8 \mathrm{~F}\right)$ considered as the two capacitances in series:

$$
C_{\text {diff }}=\frac{\left(C_{G C h} \times C_{\text {Stern }}\right)}{\left(C_{G C h}+C_{\text {Stern }}\right)}
$$


and with $C_{G C h}$ is:

$$
C_{G C h}=\sqrt{\frac{2 z^{2} q^{2} \varepsilon \varepsilon_{0} I}{k T}} \cosh \left(\frac{z q \psi_{0}}{2 k T}\right)
$$

where $z$ is the coordination number of the ions (one in the case of protons), $\varepsilon_{0}$ is the dielectric constant of vacuum, $\varepsilon$ is the relative dielectric constant of the medium (water in our case), and $I$ is the number concentration of the ions in the bulk. $\beta_{\text {int }}$ in the site-binding model depends upon the different dissociation constants and the density of binding sites:

$$
\beta_{\text {int }}=N_{S} \frac{K_{b} a_{H_{S}^{+}}^{2}+4 K_{a} K_{b} a_{H_{S}^{+}}+K_{a} k_{b}^{2}}{\left(K_{a} K_{b}+K_{b} a_{H_{S}^{+}}+a_{H_{S}^{+}}^{2}\right)^{2}} 2.3 a_{H_{S}^{+}}
$$

where $K_{i}$ are the different dissociation constants and $a_{H_{S}^{+}}$is the proton activity at the surface of the liquid interface, which is related to the bulk $a_{H_{B}^{+}}$as:

$$
a_{H_{S}^{+}}=a_{H_{B}^{+}} \exp \frac{-q \psi_{0}}{k T}
$$

or equivalently:

$$
p H_{S}=p H_{B}+\frac{q \psi_{0}}{2.3 k T}
$$

where the origin of $\psi_{0}$ is chosen at the point of zero charge. Combining Equations (3)-(6) it is also possible to extract an experimental $\beta_{\text {int }}$ and compare it with the site binding model where the $N_{s}$ used is the one calculated with our SPR results. In this model we used the values of $V_{G S}$ to calculate $\psi_{0}$ using the following relation:

$$
\psi_{0}(p H)=-\left(V_{G S}(p H)-V_{G S}\left(p H_{p z c}\right)\right)
$$

where $p H_{p z c}$ is the $\mathrm{pH}$ value at the point of zero charge $\left.\left(p H_{p z c}=p K_{a}+p K_{b}\right) / 2\right)$. The exact value of $V_{G S}\left(p H_{P Z C}\right)$ was extracted from the linear fit of the experimental $V_{G S}(p H)$.

Figure 4 shows the comparison of the experimental and the model $\beta_{\text {int }}$ with changing $\mathrm{pH}$ for all the devices (model and experimental $\beta_{\text {int }}$ are represented with black solid signs and red open signs, respectively, while device 1 to 3 are represented with squares, circles and triangles, respectively). The model of $\beta_{\text {int }}$ for APTES shows how the number of active sites that provide buffering capacitance increase for the values of $\mathrm{pH}$ lower and higher than $\sim 3.63$ and $\sim 9$, respectively, corresponding to the APTES dissociation constants for $p K_{b}$ and $p K_{a}$, respectively. The experimental $\beta_{\text {int }}$ also follows that behavior, but there is also an increase of the buffering sites at $\mathrm{pH}$ slightly higher than 7.5. This could be attributed also to contaminants or defects in the functionalization, that would contribute with charges, for example, from uncovered silanol groups (that have $p K_{a}$ at 6 ). However, the differences between the experimental $\beta_{\text {int }}$ all three devices showed the same behavior that we interpret as a similar functionalization achieved on the three sensors, which we attribute to the large surface area of all devices.

The behavior of the experimental $\beta_{\text {int }}$, shown in red in Figure 4, having increasing buffering capacitance at more than one $\mathrm{pH}$ value, is also a chemical fingerprint of the APTES functionalization. This behavior is opposed to the $\mathrm{SiO}_{2}$ where $\beta_{\text {int }}$ has only one maximum attributed to the single protonation of silanol groups (the comparison of the experimental $\beta_{\text {int }}$ from the first device is shown in Supplementary Materials). 


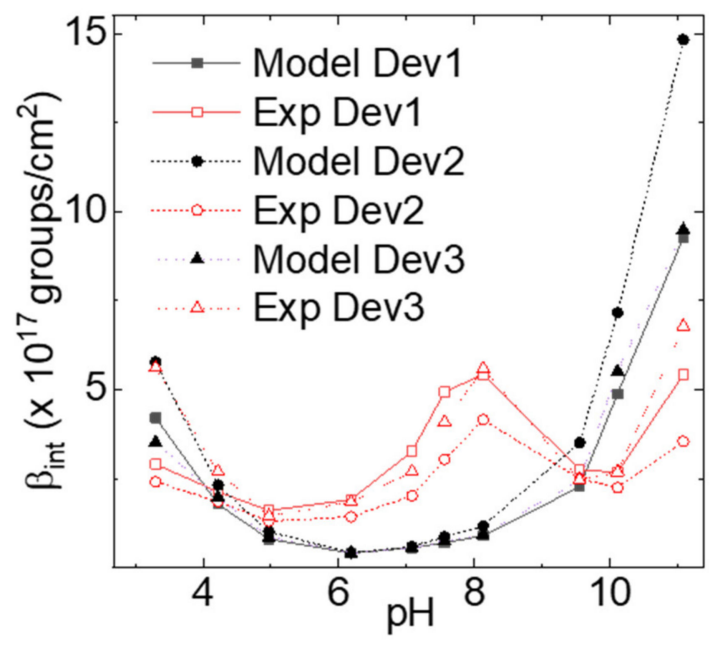

Figure 4. Comparison between modelled (in black) and experimental (in red) intrinsic buffering capacitance of the APTES functionalized surface as obtained for the three devices 1 to 3 represented with squares, circles and triangles, respectively.

\subsection{Preservation of Linear Response by the FinFETs in the Output Characteristics}

For multiplexed systems, it is also important to consider that measuring the output currents may be more convenient than following the surface potential with the reference electrode, which in most configurations will be common to all devices [20]. Previously, we showed that the high aspect ratio of FinFETs provides a more linear $\Delta I_{D S} / \Delta \mathrm{pH}$ response in comparison to Si NW-based FETs [19], while the double side gating would provide a sensitivity advantage to the FinFETs with respect to planar NWs. Figure 5 a shows $I_{d s}$ vs. $V_{d s}$ at different $\mathrm{pH}$ buffer solutions $(3,5,7,8,10)$, before and after APTES silanization. For these experiments we connected directly the source with the gate voltage $\left(V_{G S}=0\right)$. The conductance of the devices increased with increasing $\mathrm{pH}$ of the buffer solution due to the effect of the proton concentration on the majority charge carriers as described earlier. We evaluated the conductance of the FinFET devices at different buffer $\mathrm{pH}$ values before and after APTES silanization from the linear fitting of the output characteristics. The conductance of the devices increased with increase in $\mathrm{pH}$ of the buffer solution and is shown as a scatter plot before (black solid squares) and after silanization (red open circles) in Figure 5b. We observed a small bump around $15 \mathrm{mV}$, which we attribute to defects on the contacts, nevertheless the curves were symmetric and the $I_{D S}$ vs. $V_{D S}$ pass through zero, so we considered our devices Ohmic in the studied range. On linear fitting of the conductance plots shown as black and red lines in Figure $5 b$, we obtained average change in conductance of the devices as $158 \pm 9 \mathrm{nS} / \mathrm{pH}$ and $176 \pm 6 \mathrm{mS} / \mathrm{pH}$ per unit area before and after APTES silanization, respectively. We attribute the increase in conductance change of the devices to the changes in the density of $\mathrm{pH}$ sensitive groups at the oxide surface as described earlier. Moreover, the increased linear response in conductance change on APTES silanization is in accordance with the linearity increment observed in the surface potential changes. 

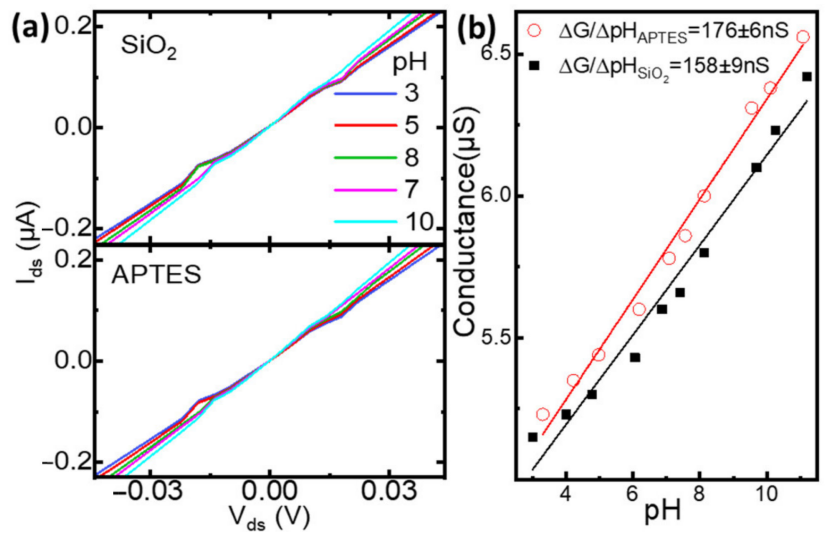

Figure 5. (a) Output characteristics of the FinFET device 1 measured before and after the APTES silanization process at different buffer $\mathrm{pH}$ values $(3,5,7,8,10)$ shown in different colors (blue, red, green pink and light blue, respectively). (b) Scatter plot showing the variation of conductance (G) in the FinFET, evaluated from the output characteristics as a function of buffer $\mathrm{pH}$ (varied from 3 to 11) before (black squares) and after the silanization process (red open circles). The linear fits of the scatter plots are shown as black and red lines for before and after APTES silanization, respectively. The average conductance change $(\Delta \mathrm{G} / \Delta \mathrm{pH})$ is also shown in the inset. For these experiments $V_{G S}=0$.

\section{Conclusions}

In this work we have presented a high height-to-width aspect ratio FinFET chemical sensor with a surface modification of the dielectric barrier using APTES coatings. We used a vapor-phase silanization method made in a home-built setup to obtain a well-defined thickness of APTES molecules linked covalently at the $\mathrm{SiO}_{2}$ sensing surface of the FinFETs. We tested the sensitivity of the FinFET devices before and after chemical modification with APTES in a buffer $\mathrm{pH}$ range of 3 to $11 . \Delta V_{G S} / \Delta \mathrm{pH}$ improved from $31 \mathrm{mV} / \mathrm{pH}$ measured on the devices with $\mathrm{SiO}_{2}$ to $36 \mathrm{mV} / \mathrm{pH}$ with APTES. In addition to the increment in the sensitivity of the FinFET devices, the linearity in the $\mathrm{pH}$ response also improved with APTES functionalization. The output response of the chemically modified high-aspect ratio FinFETs also showed an improvement in linearity and conductance change $(\Delta \mathrm{G} / \Delta \mathrm{pH})$. We attribute the enhanced $\mathrm{pH}$ sensitivity and response linearity of the chemically modified FinFET devices to the presence of amine groups at the sensing oxide, which can create charges both with protonation and deprotonation mechanisms.

The improvements we obtained were modest, probably because our starting surface was not optimum for the growth of the $\mathrm{SiO}_{2}$ and we could not use a dedicated reactor. Some authors followed the functionalization following the increase of thickness by ellipsometry [18], or with AFM measurements. We could not use AFM due to the high aspect ratio of the FinFETs, but we followed the formation of APTES layers with ellipsometry in a $\mathrm{SiO}_{2}$ substrate deposited in parallel with our wires, and in an SPR chip. We analyzed the contribution of the buffering capacitance of the different reactive groups at the sensing oxide surface that influenced the proton sensitivity of the chemically modified FinFET devices. We compared the $\beta_{\text {int }}$ evaluated from a site binding model and experimentally derived using a Stern-Gouy-Chapman approximation for the differential capacitance. The comparison of the experimental data with a model considering only amine groups showed the existence of a contribution compatible with the presence of unoccupied silanol sites. This contribution suggests also that the distribution of APTES was not completely homogeneous, and that pockets and small aggregates may have occurred in the functionalization. Thus, the $\beta_{\text {int }}$ evaluated from the experimental data is also a fingerprint of the functionalization.

In conclusion, chemical modification (with APTES) of high-aspect-ratio FinFETs which are intrinsically advantageous due to their improved linearity in transduction owing to the $2 \mathrm{D}$ depletion along the width of the device, supplements their response in terms of 
sensitivity and linearity that is desired for bio-chemical sensors. High-aspect-ratio FinFETs provide higher currents than NWs by a factor proportional to the height-to-width aspect ratio. The high-aspect-ratio FinFETs keep many of the advantages of Si NWs regarding the more efficient gating and smaller footprints, while incorporating other advantages typical of planar sensors, as there is a larger planar surface area available for functionalization and a conduction channel less sensitive to defects. Also, they offer direct gating which is not contaminated by parasitic capacitance during gate coupling, as compared to extended gate configurations. Regardless, the modest improvement in sensitivity by APTES has shown in the literature to improve the stability of $\mathrm{SiO}_{2}$ and to be a good tethering point for peptide bond functionalization. Thus, the APTES-functionalized FinFETs are promising both for $\mathrm{pH}$ sensing and for the development of biosensors using the amine sites as tethering groups for the functionalization of other receptor molecules.

Supplementary Materials: The following are available online at https:/ / www.mdpi.com/2227-904 0/9/2/20/s1, Figure S1: Full SPR spectra after APTES silanization of the SPR sensor chips at two wavelengths, 670 and $785 \mathrm{~nm}$, illustrating good alignment between the measured and the modeled data. The measured spectra are shown in light blue and red colors while the modeled one as dark blue and brown colors, Figure S2: SEM pictures and the corresponding Ids vs Vds pH curves from devices 1 to 3, Figure S3: Plot showing the variation of VGS change extracted from the transfer characteristics as a function of buffer $\mathrm{pH}$ for 3 FinFETs $(\mathrm{Ch} 1,2,3)$ measured before $(\mathrm{SiO} 2)$ and after the APTES silanization. The VGS change was extracted by subtracting the VGS values obtained after fixing the IDS, at each buffer $\mathrm{pH}$ value with respect to the value at $\mathrm{pH} 3$, Figure S4: comparison between the buffering capacitance of $\mathrm{SiO} 2$ (a) and the APTES functionalised surface in device 1.

Author Contributions: Conceptualization, C.P.G. and W.O.; methodology, C.P.G., S.K.; validation, C.P.G. and S.K.; formal analysis, C.P.G. and D.R.; investigation, C.P.G. and D.R.; resources, C.P.G. and S.K.; data curation, C.P.G., D.R. and S.R.; writing-original draft preparation, C.P.G. and D.R.; writing-review and editing All; supervision, C.P.G., S.K.; project administration, C.P.G., S.K.; funding acquisition C.P.G., S.K. All authors have read and agreed to the published version of the manuscript.

Funding: This work was financed by the H2020 program in the project ELECTROMED in the framework of the FET-OPEN Action (Grant agreement ID: 862539) and by the Luxembourg National Fund (FNR) with the projects NANOpH (grant 5718158) under the Attract program and PLASENS (grant CM15/MS/10459961) under the CORE program.

Institutional Review Board Statement: Not applicable.

Informed Consent Statement: Not applicable.

Data Availability Statement: The data presented in this study are available on request from the corresponding author.

Conflicts of Interest: The authors declare no conflict of interest.

\section{References}

1. Bergveld, P. Thirty years of ISFETOLOGY: What happened in the past 30 years and what may happen in the next 30 years. Sensors Actuators B Chem. 2003, 88, 1-20. [CrossRef]

2. Syu, Y.-C.; Hsu, W.-E.; Lin, C.-T. Review-Field-Effect Transistor Biosensing: Devices and Clinical Applications. ECS J. Solid State Sci. Technol. 2018, 7, Q3196-Q3207. [CrossRef]

3. Bergveld, P. Development of an Ion-Sensitive Solid-State Device for Neurophysiological Measurements. IEEE Trans. Biomed. Eng. 1970, 1, 70-71. Available online: https: / /ieeexplore-ieee-org.proxy.bnl.lu/stamp/stamp.jsp?tp=\&arnumber=4502688 (accessed on 19 January 2021). [CrossRef] [PubMed]

4. Rothberg, J.M.; Hinz, W.; Rearick, T.M.; Schultz, J.; Mileski, W.; Davey, M.; Leamon, J.H.; Johnson, K.; Milgrew, M.J.; Edwards, M.; et al. An integrated semiconductor device enabling non-optical genome sequencing. Nature 2011, 475, 348-352. [CrossRef] [PubMed]

5. Merriman, B.; Torrent, I.; Rothberg, J.M. Progress in Ion Torrent semiconductor chip based sequencing. Electrophoresis 2012, 33, 3397-3417. [CrossRef] 
6. Moser, N.; Keeble, L.; Rodriguez-Manzano, J.; Georgiou, P. ISFET arrays for lab-on-chip technology: A review. In Proceedings of the 2019 26th IEEE International Conference on Electronics, Circuits and Systems (ICECS), Genoa, Italy, 27-29 November 2019; pp. 57-60. [CrossRef]

7. Moser, N.; Lande, T.S.; Toumazou, C.; Georgiou, P. ISFETs in CMOS and Emergent Trends in Instrumentation: A Review. IEEE Sens. J. 2016, 16, 6496-6514. [CrossRef]

8. Kaisti, M. Detection principles of biological and chemical FET sensors. Biosens. Bioelectron. 2017, 98, 437-448. [CrossRef]

9. Cai, J.; Ning, T.H.; Yau, J.-B.; Zafar, S. Charge sensors using inverted lateral bipolar junction transistors. Google Patents US 8,980,667 B2, 17 March 2015.

10. Zaborowski, M.; Tomaszewski, D.; Dumania, P.; Grabiec, P. From FinFET to nanowire ISFET. In Proceedings of the European Solid-State Device Research Conference (ESSDERC), Bordeaux, France, 17-21 September 2012; pp. 165-168. [CrossRef]

11. Nair, P.R.; Alam, M.A. Performance limits of nanobiosensors. Appl. Phys. Lett. 2006, 88, 9-11. [CrossRef]

12. Chen, S.; Van Den Berg, A.; Carlen, E.T. Sensitivity and detection limit analysis of silicon nanowire bio(chemical) sensors. Sensors Actuators B Chem. 2015, 209, 486-489. [CrossRef]

13. Chen, S. Silicon Nanowire Field-Effect Chemical Sensor; Wohrmann Print Service: Zutphen, The Netherlands, 2011; Volume 4, pp. 915-919.

14. Rollo, S.; Rani, D.; Olthuis, W.; García, C.P. Single step fabrication of Silicon resistors on SOI substrate used as Thermistors. Sci. Rep. 2019, 9, 1-7. [CrossRef]

15. Topkar, A.; Lal, R. Effect of electrolyte exposure on silicon dioxide in electrolyte-oxide-semiconductor structures. Thin Solid Films 1993, 232, 265-270. [CrossRef]

16. Van Hal, R.E.G.; Eijkel, J.C.T.; Bergveld, P. A general model to describe the electrostatic potential at electrolyte oxide interfaces. Adv. Colloid Interface Sci. 1996, 69, 31-62. [CrossRef]

17. Chen, S.; Bomer, J.G.; Carlen, E.T.; Van Den Berg, A. $\mathrm{Al}_{2} \mathrm{O}_{3}$ / silicon nanoISFET with near ideal nernstian response. Nano Lett. 2011, 11, 2334-2341. [CrossRef] [PubMed]

18. Liang, Y.; Huang, J.; Zang, P.; Kim, J.; Hu, W. Molecular layer deposition of APTES on silicon nanowire biosensors: Surface characterization, stability and $\mathrm{pH}$ response. Appl. Surf. Sci. 2014, 322, 202-208. [CrossRef]

19. Rollo, S.; Rani, D.; Leturcq, R.; Olthuis, W.; García, C.P. High Aspect Ratio Fin-Ion Sensitive Field Effect Transistor: Compromises toward Better Electrochemical Biosensing. Nano Lett. 2019. [CrossRef]

20. Miscourides, N.; Georgiou, P. ISFET arrays in CMOS: A head-to-head comparison between voltage and current mode. IEEE Sens. J. 2019, 19, 1224-1238. [CrossRef]

21. Rollo, S.; Rani, D.; Olthuis, W.; García, C.P. High performance Fin-FET electrochemical sensor with high-k dielectric materials. Sensors Actuators B Chem. 2020, 303, 127215. [CrossRef]

22. Granqvist, N.; Liang, H.; Laurila, T.; Sadowski, J.; Yliperttula, M.; Viitala, T. Characterizing ultrathin and thick organic layers by surface plasmon resonance three-wavelength and waveguide mode analysis. Langmuir 2013, 29, 8561-8571. [CrossRef]

23. Kari, O.K.; Rojalin, T.; Salmaso, S.; Barattin, M.; Jarva, H.; Meri, S.; Yliperttula, M.; Viitala, T.; Urtti, A. Multi-parametric surface plasmon resonance platform for studying liposome-serum interactions and protein corona formation. Drug Deliv. Transl. Res. 2017, 7, 228-240. [CrossRef]

24. Zhao, H.; Brown, P.H.; Schuck, P. On the distribution of protein refractive index increments. Biophys. J. 2011, 100, 2309-2317. [CrossRef]

25. Munief, W.M.; Heib, F.; Hempel, F.; Lu, X.; Schwartz, M.; Pachauri, V.; Hempelmann, R.; Schmitt, M.; Ingebrandt, S. Silane deposition via gas-phase evaporation and high-resolution surface characterization of the ultrathin siloxane coatings. Langmuir 2018, 34, 10217-10229. [CrossRef] [PubMed]

26. Extracted from Chemical Properties of 3-Aminopropyltriethoxysilane. Available online: https://www.chemicalbook.com/ ProductMSDSDetailCB8686147_EN.htm (accessed on 19 January 2021). 